\title{
A SIMPLE SYNTHESIS OF SELINONE, AN ANTIFUNGAL COMPONENT OF MONOTES ENGLERI
}

\author{
Ágnes Kenéz, Laszló Juhász and Sándor Antus* \\ Department of Organic Chemistry, University of Debrecen, \\ P.O. Box 20, H-4010 Debrecen, Hongary
}

\begin{abstract}
A new synthesis of racemic 5,7-dihydroxy-2-[4-(3-methyl-but-2-enyloxy)-phenyl]chro . man-4-one (selinone) (rac-1a) isolated from Monotes engleri GILG. was accomplished by two routes starting from MOM-protected phloracetophenone (2).
\end{abstract}

\section{Introduction}

Recently one of us has described the isolation and structure elucidation of ( \pm )-5.7dihydroxy-2-[4-(3-methyl-but-2-enyloxy)-phenyl]chroman-4-one (rac-1a) from Monotes engleri GILG. Which shows remarkable antifungal activity against Candida albicans. 'Its levoratatory enantiomer, (-)-selinone (1a) has also been isolated from Selinum vaginatum. ${ }^{2}$ In continuation of our investigation on biologically active natural products, ${ }^{3-6}$ we report now a new synthesis of racemic selinone (rac-1a) starting from MOM-protected phloracetophenone (2).

\section{Results and discussion}

Our synthetic approach to rac-1 was based on the well-documented transformation of $2^{\prime}, 4^{\prime}, 6^{\prime}$-trihydroxychalcones into 5,7-dihydroxyflavanones under mild basic conditions. ${ }^{7,8}$ 
Therefore 1-(2-hydroxy-4,6-dimethoxymethoxyphenyl)ethanone (2) ${ }^{9}$ and 4-(3-methyl-but-2enyloxy)benzaldehyde $(3)^{10}$ were converted into the corresponding 2 '-hydroxychalcone (4a) in the presence of potassium hydroxide in ethanol with $46 \%$ yield. The deprotection $(\mathbf{4 a} \rightarrow \mathbf{4 b}$ ) under mild acidic condition $(10 \% \mathrm{HCl} / \mathrm{MeOH})$ and cyclization $(4 \mathrm{~b} \rightarrow$ rac-1a) on treatment with sodium acetate afforded racemic selinone (rac-1a) in low yield (13\%) beside its 7-methoxymethyl-ether (rac-1b) (23\%) and racemic naringenin (rac-1c) (37\%). TLC-monitoring of the first step of this transformation $(\mathbf{4 a} \rightarrow \mathbf{4 b}$ ) has clearly shown that the cleavage of the C-6' methoxymethyl group of 4a took place very rapidly to result in $\mathbf{4 c}$. However, it was followed not only by the cleavage of its methoxymethyl group, but surprisingly by its prenyl one as well. By the treatment of $4 \mathrm{a}$ with $5 \%$ $\mathrm{HCl}$ in methanol at room temperature followed on treatment with sodium acetate rac-1b could be obtained in good yield (60\%). All of our attempts to transform rac-1b to rac-1a were unsuccessful under different conditions $\left(\mathrm{SnCl}_{4}\right.$ or $\mathrm{BF}_{3}$ in dichloromethane at $-10^{\circ} \mathrm{C}$ or Amberlyst- 15 in benzene at $\left.50^{\circ} \mathrm{C}\right)$.

\section{Scheme 1.}

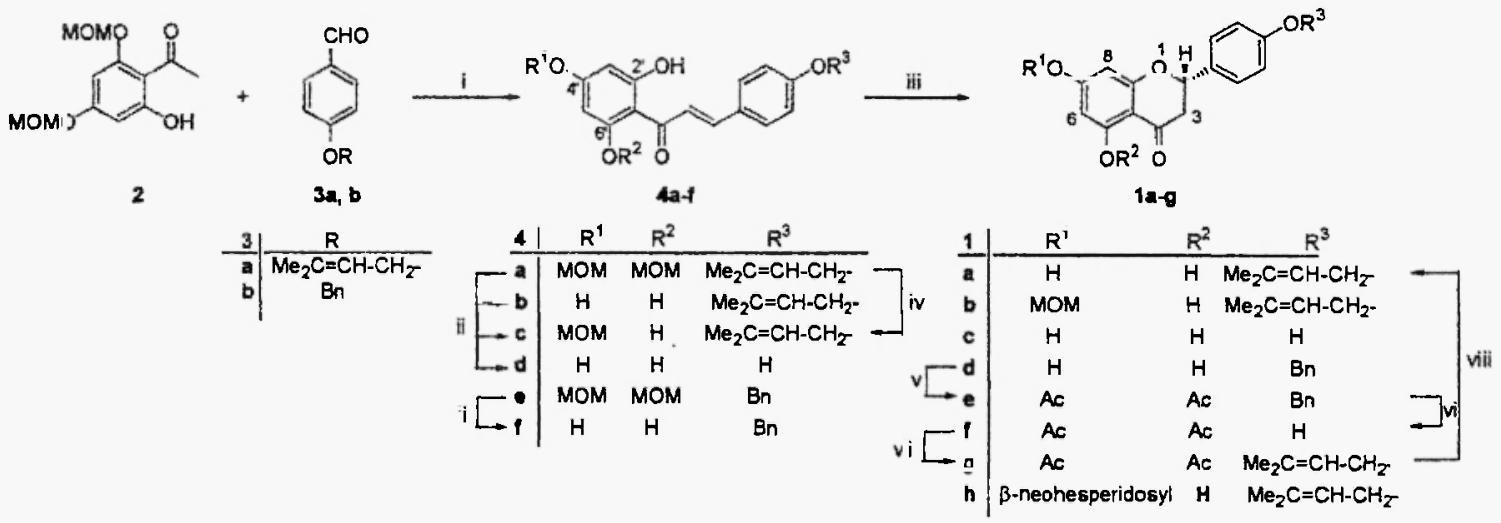

Reaction conditions: i) $\mathrm{KOH} / \mathrm{EtOH}$, rt.; ii) $10 \% \mathrm{HCl} / \mathrm{MeOH}$ reflux; iii) $\mathrm{NaOAc} / \mathrm{MeOH}$; iv) $5 \% \mathrm{HClMeOH}$, st.; v) $\mathrm{Ac}_{2} \mathrm{O} /$ dry pyridine, rt.; vi) $\mathrm{H}_{2}-\mathrm{Pd} / \mathrm{MeOH}$, rt; vii) 3-methyl-2-buten-1-ol, $\mathrm{Ph}_{3} \mathrm{P}$, diisopropyl azodicarboxylate, dry THF; viii) $\mathrm{NaOMe} / \mathrm{dry} \mathrm{MeOH}$

In order to achieve a practical route to racemic selinone (rac-1a), 5,7-diacethyl-naringenin (rac-1f) was prepared in racemic form by a simple five-step sequence starting from 2 and $3 b^{11}$ ( 2 and $\mathbf{3 b} \rightarrow \mathbf{4 e} \rightarrow \mathbf{4 f} \rightarrow$ rac-1d $\rightarrow$ rac-1e $\rightarrow$ rac-1f). Subsequently, rac-1f was prenylated by 3methyl-2-buten-1-ol under Mitsunobu condition ${ }^{12}$ to afford racemic selinone peracetate (rac-1g) whose saponification with $1 \mathrm{~N}$ sodium methoxide in methanol gave rac-1a in good yield (95\%). All spectra of rac-1a are identical with those of the natural and synthetic product prepared by Wagner et al. from $1 \mathbf{h} .^{13}$ 


\section{Experimental}

General experimental procedures: Melting points were determined on a Kofler hot stage apparatus and are uncorrected. The analytical and preparative TLC were performed on plates Kieselgel $60 \mathrm{~F}_{254}$ (Fa. Merck). The ${ }^{1} \mathrm{H}-\mathrm{NMR}$ spectra were recorded on Bruker WP-200 spectrometer with TMS as internal standard in $\mathrm{CDCl}_{3}$ and in DMSO (marked by an asterisk*). The chemical shifts are given in $\delta(\mathrm{ppm})$ and the spin-spin coupling constants $(J)$ in $\mathrm{Hz}$. HRMS were recorded in EI mode at $70 \mathrm{eV}$ on a VG $7035 \mathrm{MS}$ spectrometer. For workup the solutions were dried $\left(\mathrm{MgSO}_{4}\right)$ and concetrated in vacuo.

\section{1-(2-hydroxy-4,6-dimethoxymethoxyphenyl)-3-[4-(3-methyl-but-2-enyloxy)phenyl]-}

\section{prop-2-en-1-one (4a)}

A solution of $\mathrm{KOH}(20 \mathrm{~g})$ in water $(20 \mathrm{~mL})$ was added to the stirred solution of $2(4.23 \mathrm{~g}, 16$ $\mathrm{mmol})$ and $3 \mathrm{a}(3.14 \mathrm{~g} ; 16 \mathrm{mmol})$ in ethanol $(40 \mathrm{ml})$. The reaction mixture was stirred for 8 hours and then solvent was evaporated. The residue was diluted with water $(100 \mathrm{~mL})$, acidified with $10 \%$ $\mathrm{HCl}(\mathrm{pH}=2)$, and then the product was extracted with ethyl acetate $(3 \times 30 \mathrm{~mL})$. Organic layer was washed with water $(2 \times 10 \mathrm{~mL})$, dried and evaporated to give red solid residue whose crystallization from methanol resulted in $4 \mathrm{a}$ as orange crystals $\left(3.18 \mathrm{~g} ; 46 \%\right.$; m.p.: $\left.72-75^{\circ} \mathrm{C}\right) .{ }^{2} \mathrm{H}-\mathrm{NMR}: 1.76(3 \mathrm{H}$, $\left.\mathrm{s} ; \mathrm{CH}_{3}\right), 1.81\left(3 \mathrm{H}, \mathrm{s} ; \mathrm{CH}_{3}\right), 3.48\left(3 \mathrm{H}, \mathrm{s} ; \mathrm{OCH}_{3}\right), 3.54\left(3 \mathrm{H}, \mathrm{s} ; \mathrm{OCH}_{3}\right), 4.55\left(2 \mathrm{H}, \mathrm{d} ; J=6.7, \mathrm{CH}_{2}\right)$, $5.19\left(2 \mathrm{H}, \mathrm{s} ; \mathrm{OCH}_{2} \mathrm{O}\right), 5.29\left(2 \mathrm{H}, \mathrm{s} ; \mathrm{OCH}_{2} \mathrm{O}\right), 5.52(1 \mathrm{H}, \mathrm{m} ; \mathrm{CH}), 6.25\left(1 \mathrm{H}, \mathrm{d} ; J=2.34,3^{\prime}-\mathrm{H}\right), 6.31$ $\left(1 \mathrm{H}, \mathrm{d} ; J=2.34,5^{\prime}-\mathrm{H}\right), 6.93\left(2 \mathrm{H}, \mathrm{d} ; J=8.74,3 "-\mathrm{H}, 5^{\prime \prime}-\mathrm{H}\right), 7.55(2 \mathrm{H}, \mathrm{d} ; J=8.74,2 "-\mathrm{H}, 6 "-\mathrm{H}), 7.81$ $(2 \mathrm{H}, \mathrm{s} ; 2-\mathrm{H}, 3-\mathrm{H}), 13.95\left(1 \mathrm{H}, \mathrm{s} ; 2^{\prime}-\mathrm{OH}\right.$ ). HRMS m/z: 428.1837 (calcd for $\mathrm{C}_{24} \mathrm{H}_{28} \mathrm{O}_{7}, 428.1835$ ).

( \pm )-5,7-dihydroxy-2-[4-(3-methyl-but-2-enyloxy)phenyl]chroman-4-one (rac-1a) (racselinone) and ( \pm )-5-Hydroxy-7-methoxymetoxy-2-[4-(3-methyl-but-2-enyloxy)phenyl]chroman-4-one (rac-1b) and ( \pm )-5,7-Dihydroxy-2-(4-hydroxyphenyl)chroman-4-one (rac-1c) (racnaringenin)

To the stirred solution of $4 \mathrm{a}(0.23 \mathrm{~g} ; 0.54 \mathrm{mmol})$ in methanol $(10 \mathrm{ml}) 1 \mathrm{ml} 10 \% \mathrm{HCl}$ was added and the reaction mixture was refluxed for 2-3 hours. Subsequently $0.51 \mathrm{~g} \mathrm{NaOAc}$ was added and the stirring was continued for 3 hours. The reaction mixture was diluted with water, and extracted with ethyl acetate. The organic layer was washed with water and dried. Evaporation of the solvent gave an oil (160 mg) which was purified by column chromatography (hexane-ethyl acetate $=3: 1)$ to furnished rac-1a, $-1 \mathbf{b}$, and $-1 \mathrm{c}$. rac-1a $(24 \mathrm{mg}, 13 \%) \mathrm{m} . \mathrm{p} .143-145^{\circ} \mathrm{C} .{ }^{1} \mathrm{H}-\mathrm{NMR} * 1.71$ $\left(3 \mathrm{H}, \mathrm{s} ; \mathrm{CH}_{3}\right), 1.74\left(3 \mathrm{H}, \mathrm{s} ; \mathrm{CH}_{3}\right), 2.71\left(1 \mathrm{H}, \mathrm{dd} ; J=3.04,17.01 ; 3-\mathrm{H}_{\mathrm{eq}}\right), 3.09(1 \mathrm{H}, \mathrm{dd} ; J=12.6,17.01$; 
$\left.3-\mathrm{H}_{\mathrm{ax}}\right), 4.54\left(2 \mathrm{H}, \mathrm{d} ; J=6.64 ; \mathrm{CH}_{2}\right), 5.23(1 \mathrm{H}, \mathrm{dd} ; J=3.04,12.6 ; 2-\mathrm{H}), 5.43(1 \mathrm{H}, \mathrm{m} ; \mathrm{CH}), 5.88(2 \mathrm{H}$, s; 8-H, 6-H), $6.96\left(1 \mathrm{H}, \mathrm{d} ; J=8.7,2^{\prime}-\mathrm{H}, 66^{\prime}-\mathrm{H}\right), 7.42\left(1 \mathrm{H}, \mathrm{d} ; J=8.7,3^{\prime}-\mathrm{H}, 5^{\prime}-\mathrm{H}\right), 10.83(1 \mathrm{H}, \mathrm{s} ; 7-$ $\mathrm{OH}$ ), $12.15\left(1 \mathrm{H}, \mathrm{s} ; 5-\mathrm{OH}\right.$ ). HRMS $m / z 340.1309$ (calcd for $\mathrm{C}_{20} \mathrm{H}_{20} \mathrm{O}_{5}, 340.1311$ ). It was also obtained from rac-1g in $95 \%$ yield by treatment with $1 \mathrm{~N}$ sodium methoxyde in methanol at room temperature.

rac-1b: 50 mg (23\%, m.p.: $\left.91-93^{\circ} \mathrm{C}\right),{ }^{3} \mathrm{H}-\mathrm{NMR}: 1.75\left(3 \mathrm{H}, \mathrm{s} ; \mathrm{CH}_{3}\right), 1.80\left(3 \mathrm{H}, \mathrm{s} ; \mathrm{CH}_{3}\right), 2.78$ $\left(1 \mathrm{H}, \mathrm{dd} ; J=3.16,17.18 ; 3-\mathrm{H}_{\mathrm{eq}}\right), 3.11\left(1 \mathrm{H}, \mathrm{dd} ; J=12.96,17.18 ; 3-\mathrm{H}_{\mathrm{ax}}\right), 3.46\left(3 \mathrm{H}, \mathrm{s} ; \mathrm{OCH}_{3}\right), 4.53$ $\left(2 \mathrm{H}, \mathrm{d} ; J=6.7 ; \mathrm{CH}_{2}\right), 5.16\left(2 \mathrm{H}, \mathrm{s} ; \mathrm{OCH}_{2} \mathrm{O}\right), 5.36(1 \mathrm{H}, \mathrm{dd} ; J=3.16,12.96 ; 2-\mathrm{H}), 5.48(1 \mathrm{H}, \mathrm{m} ; \mathrm{CH})$, $6.17(1 \mathrm{H}, \mathrm{d} ; J=2.26 ; 6-\mathrm{H}), 6.20(1 \mathrm{H}, \mathrm{d} ; J=2.26 ; 8-\mathrm{H}), 6.98\left(2 \mathrm{H}, \mathrm{d} ; J=8.8 ; 2^{\prime}-\mathrm{H}, 6\right.$ ' $\left.-\mathrm{H}\right), 7.39$ (2H, d; $J=8.8,3$ "-H, 5"-H), $11.96(1 \mathrm{H}, \mathrm{s} ; 5-\mathrm{OH})$. HRMS $m / z 384.1574$ (calcd for $\mathrm{C}_{22} \mathrm{H}_{24} \mathrm{O}_{6}, 384.1573$ ).

rac-1c: $(55 \mathrm{mg}, 37 \%)$ m.p.: $244-246^{\circ} \mathrm{C}$. 'H-NMR': $2.66\left(1 \mathrm{H}, \mathrm{dd} ; J=2.56,17.14,3-\mathrm{H}_{\text {eq }}\right.$ ), $3.26\left(1 \mathrm{H}, \mathrm{dd} ; J=12.84,17.14,3-\mathrm{H}_{\mathrm{ax}}\right), 5.43(1 \mathrm{H}, \mathrm{dd} ; J=2.56,12.84,2-\mathrm{H}), 5.87(2 \mathrm{H}, \mathrm{s} ; 6-\mathrm{H}, 8-\mathrm{H})$, $6.81\left(2 \mathrm{H}, \mathrm{d} ; J=8.48,2^{\prime}-\mathrm{H}, 6^{\prime}-\mathrm{H}\right), 7.33\left(2 \mathrm{H}, \mathrm{d} ; J=8.48,3^{\prime}-\mathrm{H}, 5^{\prime}-\mathrm{H}\right), 9.60\left(1 \mathrm{H}, \mathrm{s} ; 4^{\prime}-\mathrm{OH}\right), 10.8(1 \mathrm{H}$, $s ; 7-\mathrm{OH}), 12.15(1 \mathrm{H}, \mathrm{s} ; 5-\mathrm{OH})$. HRMS $m / z 272.0689$ (calcd for $\mathrm{C}_{15} \mathrm{H}_{12} \mathrm{O}_{5}, 272.0685$ ).

\section{( $)$-5-Hydroxy-7-methoxymetoxy-2-[4-(3-methyl-but-2-enyloxy)phenyl]chroman-4-one} (rac-1b)

To the stirred solution of $4 \mathrm{a}(0.23 \mathrm{~g} ; 0.54 \mathrm{mmol})$ in methanol $(50 \mathrm{ml}) 2 \mathrm{ml} 5 \% \mathrm{HCl}$ was added and the reaction mixture was stirred at room temperature for 8 hours. Subsequently $0.51 \mathrm{~g}$ $\mathrm{NaOAc}$ was added and the stirring was continued at $50^{\circ} \mathrm{C}$ for 3 hours. The reaction mixture was diluted with water, and extracted with ethyl acetate. The organic layer was washed with water and dried. Evaporation of the solvent gave an oil $(200 \mathrm{mg})$ which was purified by column chromatography (hexane-ethyl acetate $=3: 1$ ) to furnished rac-1 b as white crystalline solid (115 mg, $60 \%$, m.p.: $91.5-93^{\circ} \mathrm{C}$ ).

\section{1-(2-hydroxy-4,6-dimethoxymethoxyphenyl)-3-(4-benzyloxyphenyl)-prop-2-en-1-one} (4e)

A solution of $\mathrm{KOH}(5 \mathrm{~g})$ in water $(50 \mathrm{~mL})$ was added to the stirred solution of $2(1.82 \mathrm{~g} ; 7.1$ $\mathrm{mmol})$ and $3 \mathrm{~b}(1.5 \mathrm{~g} ; 7.1 \mathrm{mmol})$ in ethanol $(100 \mathrm{ml})$. The reaction mixture was stirred for 5 days at $40^{\circ} \mathrm{C}$. The solvent was evaporated and the residue was diluted with water $(100 \mathrm{~mL})$, acidified with $10 \% \mathrm{HCl}(\mathrm{pH}=2)$, and the product was extracted with ethyl acetate $(3 \times 30 \mathrm{~mL})$. Organic layer was washed with water $(2 \times 10 \mathrm{~mL})$, dried. Evaporation of the solvent gave an an oil $(3.16 \mathrm{~g})$, which was purified by column chromatography to furnished $4 \mathrm{e}$ as yellow crystals $(1.74 \mathrm{~g}, 54 \%$; m.p.: $115-117$ 
${ }^{\circ} \mathrm{C} .{ }^{1} \mathrm{H}-\mathrm{NMR}: 3.48\left(3 \mathrm{H}, \mathrm{s} ; \mathrm{OCH}_{3}\right), 3.53\left(3 \mathrm{H}, \mathrm{s} ; \mathrm{OCH}_{3}\right), 5.11\left(2 \mathrm{H}, \mathrm{s} ; \mathrm{OCH}_{2} \mathrm{O}\right), 5.19\left(2 \mathrm{H}, \mathrm{s} ; \mathrm{OCH}_{2} \mathrm{O}\right)$, $5.29\left(2 \mathrm{H}, \mathrm{s} ; \mathrm{CH}_{2} \mathrm{Ph}\right), 6.24\left(1 \mathrm{H}, \mathrm{d} ; J=2.28,3^{\prime}-\mathrm{H}\right), 6.31\left(1 \mathrm{H}, \mathrm{d}, J=2.28,5^{\prime}-\mathrm{H}\right) 7.0(2 \mathrm{H}, \mathrm{d} ; J=8.72$, 2"-H, 6"-H), 7.39 (5H, m; Ph), 7.56 (2H, d; $J=8.74,3 "-\mathrm{H}, 5 "-\mathrm{H}), 7.75(1 \mathrm{H}, \mathrm{d}, J=16.85,3-\mathrm{H}), 7.85$ $(1 \mathrm{H}, \mathrm{d}, J-16.85,2-\mathrm{H}) 13.92\left(1 \mathrm{H}, \mathrm{s} ; 2^{\prime}-\mathrm{OH}\right)$. HRMS $m / z 450.1676$ (calcd for $\mathrm{C}_{26} \mathrm{H}_{26} \mathrm{O}_{7}, 450.1679$ ).

\section{(士)-5,7-Dihydroxy-2-(4-benzyloxyphenyl)chroman-4-one (rac-1d)}

A solution of $4 \mathrm{e}(1.7 \mathrm{~g} ; 3.7 \mathrm{mmol})$ and $10 \% \mathrm{HCl}(5.7 \mathrm{ml})$ in methanol $(300 \mathrm{ml})$ was stirred over night at room temperature. Subsequently $3.69 \mathrm{~g} \mathrm{NaOAc}$ was added, and stirring was continued for 5 hours at $70^{\circ} \mathrm{C}$. After evaporation of the solvent the residue was diluted with water, and the product was extracted with ethyl acetate. The organic layer was washed with water, dried and evaporated to give an oil whose purification by column chromatography (hexane-ethyl acetate $=$ $3: 1)$ resulted in rac-1d $(0.60 \mathrm{~g} ; 43.9 \%)$ as a yellow crystals. mp: $219-222{ }^{\circ} \mathrm{C}(\mathrm{MeOH}) .{ }^{1} \mathrm{H}$ NMR": $2.71\left(1 \mathrm{H}, \mathrm{dd}, J=3.17,3-\mathrm{H}_{\mathrm{eq}}\right), 3.25\left(1 \mathrm{H}, \mathrm{dd}, J=4.5,12.42,3-\mathrm{H}_{\mathrm{ex}}\right), 5.17\left(2 \mathrm{H}, \mathrm{s}, \mathrm{OCH}_{2}\right), 5.5(1 \mathrm{H}$, dd, $J=2.6,12.36,2-\mathrm{H}), 7.03(1 \mathrm{H}, \mathrm{s}, 6-\mathrm{H}), 7.08(1 \mathrm{H}, \mathrm{s}, 8-\mathrm{H}) 7.28-7.46(9 \mathrm{H}, \mathrm{m}, \mathrm{Ar}-\mathrm{H}), 10.84(1 \mathrm{H}, \mathrm{s}$, 7-OH), 12.14 (1H, s, 5-OH). HRMS $m / z 362.1158$ (calcd for $\mathrm{C}_{22} \mathrm{H}_{18} \mathrm{O}_{5}, 362.1154$ ).

\section{(士)-5,7-Diacethoxy-2-(4-benzyloxyphenyl)chroman-4-one (rac-1e)}

A solution of $1 \mathrm{~d}(600 \mathrm{mg} ; 1.66 \mathrm{mmol})$ and acetic aṇhydride $(5 \mathrm{~mL})$ in dry pyridine $(20 \mathrm{ml})$ was stirred for 2 days at room temperature. After the usual workup the precipitated product was filtered off, washed with water and dried, then crystallized from methanol gave rac-1e as yellow crystals (300 mg; 50\%) mp.: 97-101 ${ }^{\circ} \mathrm{C}(\mathrm{MeOH}) .{ }^{\mathrm{I}} \mathrm{H}-\mathrm{NMR}: 2.28(3 \mathrm{H}, \mathrm{s}, \mathrm{OAc}), 2.38(3 \mathrm{H}, \mathrm{s}, \mathrm{OAc})$, $2.73\left(1 \mathrm{H}, \mathrm{dd}, J=2.8,16.64,3-\mathrm{H}_{\mathrm{eq}}\right), 3.06\left(1 \mathrm{H}, \mathrm{dd}, J=13.45,16.68,3-\mathrm{H}_{\mathrm{ax}}\right), 5.08\left(2 \mathrm{H}, \mathrm{s}, \mathrm{OCH}_{2}\right), 5.42$ $(1 \mathrm{H}, \mathrm{dd}, J=2.72,13.4,2-\mathrm{H}), 6.52(1 \mathrm{H}, \mathrm{d}, J=2.05,6-\mathrm{H}), 6.76(1 \mathrm{H}, \mathrm{d}, J=1.6,8-\mathrm{H}), 7.02(2 \mathrm{H}, \mathrm{d}, J$ $=11.3$, Ar-H), 7.39 (7H, m, Ar-H). HRMS $m / z 446.1368$ (calcd for $\mathrm{C}_{26} \mathrm{H}_{22} \mathrm{O}_{7}, 446.1366$ ).

\section{( \pm )-5,7-Diacethoxy-2-(4-hydroxyphenyl)chroman-4-one (rac-1f)}

A solution of $1 \mathrm{e}(250 \mathrm{mg}, 0.56 \mathrm{mmol})$ in dry methanol $(100 \mathrm{~mL})$ was hydrogenated in the presence of $\mathrm{Pd} / \mathrm{C}(120 \mathrm{mg})$ at r.t. The usual workup gave rac-1f $(152 \mathrm{mg}, 76 \%)$ as a yellow solid of m.p.: 118-121 ${ }^{\circ} \mathrm{C} .{ }^{1} \mathrm{H}-\mathrm{NMR}: 2.28(3 \mathrm{H}, \mathrm{s}, \mathrm{OAc}), 2.37(3 \mathrm{H}, \mathrm{s}, \mathrm{OAc}), 2.67(1 \mathrm{H}, \mathrm{dd}, J=2.56,16.45,3-$ $\left.\mathrm{H}_{\text {eq }}\right), 3.02\left(1 \mathrm{H}, \mathrm{dd}, J=13.52,16.45,3-\mathrm{H}_{\mathrm{ax}}\right), 5.31(1 \mathrm{H}, \mathrm{dd}, J=2.56,13.52,2-\mathrm{H}), 6.53(1 \mathrm{H}, \mathrm{d}, J=$ 2.19, 6-H), $6.74(1 \mathrm{H}, \mathrm{d}, J=2.19,8-\mathrm{H}), 6.82\left(2 \mathrm{H}, \mathrm{d}, J=8.4,2^{\prime}-\mathrm{H}, 6^{\prime}-\mathrm{H}\right), 7.23$ (2H, d, $J=8.4,3^{\prime}-\mathrm{H}^{\prime}$, 5'-H). HRMS $m / z 356.0891$ (calcd for $\mathrm{C}_{19} \mathrm{H}_{16} \mathrm{O}_{7}, 356.0896$ ). 
( \pm )-5,7-Diacethoxy-2-[4-(3-methyl-but-2-enyloxy)phenyl]chroman-4-one (rac-1g)

To a solution of diacetate $1 \mathrm{f}(120 \mathrm{mg}, 0.34 \mathrm{mmol})$, triphenylphosphine (107 $\mathrm{mg}, 0.41 \mathrm{mmol})$ and 3-methyl-2-buten- $1-\mathrm{ol}(50 \mu \mathrm{L}, 50 \mathrm{mmol})$ in dry THF $(10 \mathrm{~mL})$ cooled to $0^{\circ} \mathrm{C}$ was added dropwise over $30 \mathrm{~min}$ under argon a solution of diisoprophyl azodicarboxylate $(85 \mu \mathrm{L}, 55 \mathrm{mmol})$ in dry THF $(5 \mathrm{~mL})$. The reaction mixture was allowed to warm to room temperature and stirred for 2 hours. After removal of the solvent in vacuo, the residue was purified by column chromatography (hexane-ethyl acetate $=2: 1)$ to give rac-1g as white crystals of m.p.: $71.5-72^{\circ} \mathrm{C}(86 \mathrm{mg}, 60 \%) .1 \mathrm{H}$ NMR: $\delta: 1.76\left(3 \mathrm{H}, \mathrm{s} ; \mathrm{CH}_{3}\right), 1.81\left(3 \mathrm{H}, \mathrm{s} ; \mathrm{CH}_{3}\right), 2.30(3 \mathrm{H}, \mathrm{s}, \mathrm{OAc}), 2.39(3 \mathrm{H}, \mathrm{s}, \mathrm{OAc}), 2.74(1 \mathrm{H}, \mathrm{dd}$; $\left.J=2.56,16.45 ; 3-\mathrm{H}_{\mathrm{eq}}\right), 3.09\left(1 \mathrm{H}, \mathrm{dd} ; J=13.16,16.45 ; 3-\mathrm{H}_{\mathrm{ax}}\right), 4.54\left(2 \mathrm{H}, \mathrm{d} ; J=6.58 ; \mathrm{CH}_{2}\right), 5.44$ $(1 \mathrm{H}, \mathrm{dd} ; J=2.56,13.16 ; 2-\mathrm{H}), 5.50(1 \mathrm{H}, \mathrm{m} ; \mathrm{CH}), 6.53(1 \mathrm{H}, \mathrm{d}, J=2.19 ; 5-\mathrm{H}), 6.77(1 \mathrm{H}, \mathrm{d}, J=2.19$, 6-H), $6.97\left(2 \mathrm{H}, \mathrm{d}, J=11.33,2^{\prime \prime}-\mathrm{H}, 6^{\prime \prime}-\mathrm{H}\right), 7.37\left(2 \mathrm{H}, \mathrm{d}, J=11.33,3^{\prime}-\mathrm{H}, 5^{\prime}-\mathrm{H}\right)$. HRMS $m / z 424.1524$ (calcd for $\mathrm{C}_{24} \mathrm{H}_{24} \mathrm{O}_{7}, 424.1522$ ).

Acknowledgement: The authors thank the National Science Foundation (OTKA T-034250) for valuable financial support.

\section{References}

1. E. Garo, J-L. Wolfender, K. Hostettmann, W. Hiller, S. Antus, S. Mavi; Helvetica Chimica Acta; 81, 754 (1998).

2. T.R. Seshadri, M.S. Sood; Tetrahedron Lett., 853 (1967)

3. L. Juhász, L. Kürti, S. J.A.C.S. J. Nat. Prod, 63, 866 (2000).

4. L. Juhász, Z. Dinya, T.E. Gunda and S. Antus; Tetrahedron Lett., 41, 2491 (2000).

5. L. Juhász, Z. Dinya, S. Antus, and T. Gunda;. Z. Naturforsch B, 56b, 6, 554 (2001).

6. K. Gulacsi; G. Litkei; S. Antus; T.E. Gunda; Tetrahedron, 54, 13867 (1998).

7. S. Antus, O. Seligmann, H. Wagner; Liebigs Ann. Chem., 647 (1986)

8. S. Antus; E. Baitz-Gacs; A. Gottsegen; T. Kovács; K. Szunyog; T.S. Toth; H. Wagner; Liebigs Ann. Chem., 105 (1993).

9. T. Patonay, D. Molnár, Z. Murányi, Bull. Soc. Chim. Fr., 132, 233 (1995).

10. V.M. Chari; G. Aurnhammer, H. Wagner; Tetrahedron Lett., 35, 3079 (1970)

11. A. Arai, I. Ichikizaki, Bull. Chem. Soc. Jpn., 35, 504 (1962).

12. S. Gester, P. Metz, O. Zierau, G. Vollmer; Tetrahedron, 57; 6; 1015 (2001).

13. G. Aurnhammer, V. M. Chari, H. Wagner; Chem. Ber., 105, 3511 (1972).

Received on June 6, 2002 